\title{
Prophylactic extended-field irradiation with concurrent chemotherapy for pelvic lymph node-positive cervical cancer
}

\author{
Jinju Oh, MD¹, Ki Ho Seol, MD, PhD², Hyun Joo Lee, MD¹, Youn Seok Choi, MD, PhD', \\ Ji Y. Park, MD' ${ }^{3}$ Jin Young Bae, MD \\ Departments of ${ }^{1}$ Obstetrics and Gynecology, ${ }^{2}$ Radiation Oncology, and ${ }^{3}$ Pathology, Catholic University of Daegu School of \\ Medicine, Daegu, Korea
}

Purpose: This study aimed to evaluate whether prophylactic extended-field pelvic radiotherapy (EF-PRT) yields better results than standard whole pelvic radiotherapy (WPRT) in patients with pelvic lymph node-positive cervical cancer treated with concurrent chemoradiotherapy (CCRT).

Materials and Methods: A total of 126 cases of stage IB-IVA cervical cancer that had pelvic lymph node involvement in magnetic resonance imaging and were treated with CCRT between 2000 and 2016 were reviewed. None of the patients had paraaortic lymph node (PALN) metastases. The patients were classified to two groups, namely, those treated with EF-PRT, including prophylactic para-aortic radiotherapy, and those treated only with WPRT. The median dose to the PALN area in patients treated with EF-PRT was $45 \mathrm{~Gy}$. All patients received concurrent cisplatin-based chemotherapy.

Results: Overall, 52 and 74 patients underwent EF-PRT and WPRT, respectively. Patient characteristics and irradiated dose were not significantly different, except the dose to the para-aortic area, between the two groups. The median follow-up period was 75.5 months (range, 5 to 195 months). The 10-year cumulative recurrence rate of PALN for EF-PRT vs. WPRT was $6.9 \%$ and $10.1 \%$ ( $p=$ 0.421), respectively. The 10-year disease-free survival and overall survival for EF-PRT vs. WPRT were $69.7 \%$ vs. $66.1 \%$ ( $p=0.748$ ) and $71.7 \%$ vs. $72.3 \%(p=0.845)$, respectively. Acute gastrointestinal complications were significantly higher in EF-PRT $(n=21$; 40.4\%) than WPRT ( $n=26 ; 35.1 \%)(p=0.046)$. Late toxicities were not significantly different in both groups.

Conclusion: In this study, prophylactic radiotherapy for PALN does not have an additional benefit in patients with pelvic lymph node-positive cervical cancer treated with CCRT.

Keywords: Uterine cervical neoplasm, Lymph node, Radiotherapy, Survival rate

\section{Introduction}

Although the status of the pelvic lymph node (LN) does not affect the International Federation Gynecology and Obstetrics (FIGO) clinical stage of cervical cancer, pelvic LN involvement is known to be among the most important adverse prognostic factors [1-4]. In invasive cervical cancer, lymphatic spread typically occurs in a stepwise progression. The pattern of nodal metastasis tends to be orderly in a contiguous and predictable manner, starting from the lower pelvis to the upper pelvis, followed by the para-aortic lymph node (PALN) $[5,6]$. The regional pelvic $L N$ becomes involved before the common iliac and para-aortic nodal chain. PALN metastases rarely occur in the absence of pelvic LN metastases. Although cervical cancer

Received 10 July 2017, Revised 19 August 2017, Accepted 01 September 2017.

Correspondence: Ki Ho Seol, MD, PhD, Department of Radiation Oncology, Catholic University of Daegu School of Medicine, 33 Duryugongwon-ro 17-gil, Nam-gu, Daegu 42472, Korea. Tel: +82-53-650-4788, Fax: +82-53-289-2697, E-mail: khseol@cu.ac.kr

(c) This is an Open Access article distributed under the terms of the Creative Commons Attribution Non-Commercial License (http://creativecommons.org/ licenses/by-nc/4.0/) which permits unrestricted non-commercial use, distribution, and reproduction in any medium, provided the original work is properly cited.

www.e-roj.org 
can metastasize to the common iliac and PALNs directly via the posterior cervical trunk, this pattern of spread is uncommon $[7,8]$. Thus, patients with pelvic LN involvement are at risk for occult PALN metastasis.

In surgical series, clinically occult PALN involvement has been reported in up to $29 \%$ of patients [9] and is more common in those with pelvic LN metastases [7]. Surgical LN staging of locally advanced cervical cancer shows that the overall $12 \%$ rate of occult PALN metastasis increases to $22 \%$ in those with pelvic LN involvement [10]. A phase III randomized trial reported that elective PALN radiotherapy can reduce paraaortic recurrence in these patients and improve survival $[11,12]$. As such, prophylactic extended-field pelvic radiotherapy (EFPRT), including the para-aortic region, has been suggested to treat occult metastases in locally advanced cervical cancer, particularly in patients with pelvic LN involvement. The rationale for EF-PRT is to sterilize micrometastatic disease and mitigate the risk of distant relapse.

However, most evidence supporting the clinical benefit of EF-PRT was derived from patients treated with radiotherapy (RT) alone before the era of platinum-based concurrent chemoradiotherapy (CCRT). The benefit of EF-PRT is unclear in concurrent platinum-based chemotherapy. As such, we conducted a retrospective study to determine the benefit of EF-PRT in patients with cervical cancer with pelvic LN involvement. This study aimed to evaluate whether EF-PRT yields better results than standard whole pelvic radiotherapy (WPRT) in patients with pelvic LN-positive cervical cancer treated with CCRT.

\section{Materials and Methods}

\section{Patients}

We retrospectively reviewed 310 cases of cervical cancer treated between 2000 and 2016 using definitive CCRT with a curative aim in our institution between 2000 and 2016. The inclusion criteria were as follows: (1) newly diagnosed histologically proven squamous cell carcinoma, adenocarcinoma, or adenosquamous carcinoma of the uterine cervix; (2) clinical and radiologic FIGO stage IB-IVA with no other evidence of distant metastasis; (3) positive pelvic $\mathrm{LN}$ involvement on computed tomography (CT), magnetic resonance imaging $(\mathrm{MRI})$, or fluorodeoxyglucose positron emission tomography-computed tomography (PET/CT) scan; (4) no evidence of PALN metastasis on imaging (MRI and PET/ CT scan); and (5) treatment using pelvic RT with platinumbased concurrent chemotherapy. The LNs were classified as metastatic based on radiographic findings $(>1.0 \mathrm{~cm}$ in the short axis dimension or presence of hypermetabolic activity) at the time of diagnosis or radiological and oncological interpretation of the appearance of the nodes. The PALN and pelvic LN were not surgically assessed. Patients with a history of hysterectomy, other malignancy, prior abdominal or pelvic RT, prior chemotherapy, or positive PALN metastasis diagnosed via imaging or biopsy were excluded.

Finally, 126 patients were enrolled for analysis and were divided into two groups: WPRT (i.e., RT of the entire pelvis) and EF-PRT (i.e., RT of the entire pelvis and PALN area for prophylaxis) (Table 1).

\section{Chemoradiotherapy}

All patients were scheduled to receive combined external beam radiotherapy (EBRT) and intracavitary brachytherapy (ICBT). EBRT was delivered with four fields (anteroposterior, posteroanterior, and two lateral fields) of 6-15 MV photons or with anteroposterior-posteroanterior opposed beams of 10-15 MV photons. The decision to use EF-PRT was at the discretion of the radiation oncologist, balancing the risk of occult paraaortic metastases against the potential for increased acute and late toxicity. Patients who had a common iliac LN metastasis were treated with EF-PRT or with WPRT that fully covered the common iliac nodal area just below aortic bifurcation. The superior border of EBRT was the L3-L4 $(n=20)$ or L4-L5 ( $n=$ 54 ) interspace depending on the location of aortic bifurcation or the presence of extensive pelvic LN involvement for WPRT. In EF-PRT, the superior border was extended to encompass PALN area according to the discretion of the radiation oncologist as follows: T12-L1 $(n=9), \mathrm{L} 1-\mathrm{L} 2(n=2)$, or L2-L3 $(n=41)$ interspace. In boost RT for enlarged LN area, three-dimensional conformal RT or intensity-modulated radiotherapy (IMRT) is used since 2009. All patients received a median EBRT dose of $45 \mathrm{~Gy}$ (range, 39.6 to $54 \mathrm{~Gy}$ ) at 1.7 Gy or $1.8 \mathrm{~Gy}$ per fraction to the whole pelvic area and the boost RT of 9 Gy (range, 0 to 23.4 Gy) given at 1.8 Gy or 2 Gy per fraction to enlarged $\mathrm{LN}$ regions, involved parametrium, or involved regions of the pelvic side wall. The median dose to the PALN area in patients treated with EF-PRT was 45 Gy (range, 41.4 to 45.9 Gy) given at 1.7 or $1.8 \mathrm{~Gy}$ per fraction.

After adequate tumor regression, high-dose-rate ICBT was performed twice per week using an iridium-192 remote after-loading technique. The standard prescribed dose for each brachytherapy in our institution was $5.0 \mathrm{~Gy}$ to A-point in 6 fractions, twice weekly. The prescribed A-point dose was median 30 Gy (range, 25 to $36 \mathrm{~Gy}$ ). The combined total dose 
Table 1. Patients' characteristics

\begin{tabular}{|c|c|c|c|}
\hline Variable & $\begin{array}{l}\text { Whole pelvic radiotherapy } \\
\qquad(\mathrm{n}=74)\end{array}$ & $\begin{array}{l}\text { Extended-field pelvic radiotherapy } \\
\qquad(\mathrm{n}=52)\end{array}$ & p-value \\
\hline Age (yr) & $54.5(23-76)$ & $48(32-81)$ & 0.066 \\
\hline Primary tumor diameter (mm) & $41(10-70)$ & $46(10-72)$ & 0.112 \\
\hline Metastatic pelvic LN size ${ }^{\text {a) }}(\mathrm{mm})$ & $11.7(6-53)$ & $13(6-31.1)$ & 0.501 \\
\hline$<10$ & 18 & 11 & 0.909 \\
\hline$\geq 10$ and $<20$ & 47 & 34 & \\
\hline$\geq 20$ & 9 & 7 & \\
\hline Metastatic pelvic LN number & $2(1-6)$ & $2(1-7)$ & 0.130 \\
\hline \multicolumn{4}{|l|}{ Stage } \\
\hline |B1 & 16 & 5 & 0.411 \\
\hline IB2 & 8 & 10 & \\
\hline$\| A 1$ & 3 & 1 & \\
\hline$\| \mathrm{A} 2$ & 2 & 3 & \\
\hline$\| \mathrm{B}$ & 25 & 17 & \\
\hline IIIB & 16 & 14 & \\
\hline IVA & 4 & 2 & \\
\hline \multicolumn{4}{|l|}{ Pathologic type } \\
\hline $\mathrm{SCC}$ & 63 & 46 & \\
\hline Adenocarcinoma or ASCC & 11 & 6 & \\
\hline \multicolumn{4}{|l|}{ Differentiation } \\
\hline Well & 1 & 1 & 0.433 \\
\hline Moderately & 70 & 46 & \\
\hline Poorly & 3 & 5 & \\
\hline Pre-treatment SCC antigen level (ng/mL) & $6.48(0.5-65.29)$ & $7.4(0.1-143)$ & 0.989 \\
\hline \multicolumn{4}{|l|}{ Chemotherapy } \\
\hline Weekly cisplatin & 48 & 31 & 0.364 \\
\hline Cisplatin + 5-FU & 16 & 9 & \\
\hline Cisplatin + paclitaxel & 10 & 12 & \\
\hline
\end{tabular}

Values are presented as median (range) or number.

LN, Iymph node; SCC, squamous cell carcinoma; ASCC, adenosquamous cell carcinoma; 5-FU, 5-fluorouracil.

a) The size of the metastatic pelvic LN was evaluated via CT and MRI, maximum short-axis dimension of metastatic LN.

Table 2. Prescribed dose for the target volume (unit: cGy)

\begin{tabular}{lccc}
\hline & $\begin{array}{c}\text { Whole pelvic radiotherapy } \\
(\mathrm{n}=74)\end{array}$ & $\begin{array}{c}\text { Extended field pelvic radiotherapy } \\
(\mathrm{n}=52)\end{array}$ & $p$-value \\
\hline Prophylactically prescribed EBRT EQD2 to PALN area & 0 & $4,419.6 \pm 56.0$ & $<0.001$ \\
Prescribed EBRT EQD2 to uninvolved pelvic LN area & $4,423.9 \pm 193.7$ & $4,516.1 \pm 331.9$ & 0.076 \\
Prescribed EBRT EQD2 to enlarged pelvic LN area & $5,262.6 \pm 463.8$ & $5,234.3 \pm 343.7$ & 0.709 \\
Prescribed EBRT EQD2 to cervical mass & $4,783.9 \pm 592.3$ & $4,832.6 \pm 531.7$ & 0.636 \\
Prescribed brachytherapy EQD2 for A-point & $3,711.5 \pm 241.1$ & $3,600.7 \pm 582.1$ & 0.200 \\
Total Prescribed A-point EQD2 (= EBRT + ICR) & $8,495.4 \pm 649.5$ & $8,433.3 \pm 814.4$ & 0.636 \\
\hline
\end{tabular}

Values are presented as mean \pm standard deviation.

EBRT, external beam radiotherapy; EQD2, radiobiological equivalent dose in 2 Gy per fraction $(\alpha / \beta=10)$; PALN, para-aortic lymph node; LN, lymph node.

from EBRT and ICBT was calculated using a linear quadratic model to determine the radiobiological equivalent dose in 2 Gy fractions (EOD2) $(\alpha / \beta=10)$ [13]. The mean total prescribed A-point EQD2 was $84.98 \mathrm{~Gy}$. The prescribed dose for the target volume is given Table 2. The median overall irradiated time was 59 days (range, 45 to 133 days).

Cisplatin-based chemotherapy was delivered concurrently with EBRT in all patients. During RT, chemotherapy with 
weekly cisplatin ( $40 \mathrm{mg} / \mathrm{m}^{2}$ weekly for 6 weeks) was given to 79 patients. During EBRT, two cycles of cisplatin-based combination chemotherapy with cisplatin plus 5-fluorouracil (5-FU) or cisplatin plus paclitaxel at 3-week intervals were given to 25 and 22 patients, respectively. Chemotherapy with cisplatin and 5-FU consisted of an intravenous infusion of 75 $\mathrm{mg} / \mathrm{m}^{2}$ of cisplatin (day 1) followed by an intravenous infusion of $4,000 \mathrm{mg} / \mathrm{m}^{2}$ of 5 -FU over a 96 -hour period (days 2-5). A total of $1 \mathrm{~L}$ of normal saline was given both before and after cisplatin administration, and mannitol was used to increase urine output (day 1). Meanwhile, chemotherapy with cisplatin plus paclitaxel consisted of an intravenous infusion of $135 \mathrm{mg} / \mathrm{m}^{2}$ of paclitaxel (day 1) followed by an intravenous infusion of $75 \mathrm{mg} / \mathrm{m}^{2}$ of cisplatin (day 2).

Chemotherapy was started at the commencement of RT (day 1). Premedications included ondansetron and dexamethasone to reduce nausea and vomiting. If patients had grade 3 or 4 leukopenia or neutropenia, then granulocyte-macrophage colony-stimulating factor was used. The chemotherapeutic dose was reduced when patients had severe hematologic or non-hematologic toxicities. Chemotherapy was stopped when severe toxicities were persistent after dose reduction, when patients had poor performance status, or when patients refused the treatment.

\section{Response evaluation and follow-up}

All patients were subjected to routine post-CCRT surveillance with physical examination, cervicovaginal cytology, laboratory test (e.g., squamous cell carcinoma antigen), and imaging studies, including abdominopelvic CT, MRI, and PET/CT. After completion of CCRT, the patients were evaluated every 3 months for the first 2 years and every 6 months thereafter. Recurrence was diagnosed through physical examination and diagnostic imaging (contrast-enhanced CT, MRI, and PET/CT scans) and was confirmed histologically via needle aspiration or excisional biopsy when possible. Patients were assessed for toxicity using the Common Terminology Criteria for Adverse Events version 4.0.

\section{End points and statistical methods}

The primary end point for treatment comparison was 5- and 10-year PALN recurrence rate, disease-free survival (DFS) rate, and overall survival (OS) rate. DFS was defined as survival without locoregional, PALN, or distant recurrences. In-field pelvic recurrence was defined as recurrent or progression of primary tumor, or pelvic (regional) LN failure in the irradiated pelvic region. We considered persistent cervical disease or enlarged pelvic LN that did not regress for 3 months after completion of RT as in-field pelvic recurrence. Distant metastasis was defined as recurrence outside the infrarenal para-aortic field. For disease event, we considered the first sites of recurrence as disease sites. We calculated all occurrences from the date of diagnosis to the date of relapse or the last date of follow-up. Deaths from other cause were censored at the time of last follow-up.

The chi-square test and Fisher exact test were used to analyze the differences between the groups with respect to several prognostic factors. The survival analysis was based on the Kaplan-Meier method, and the results were compared using the log-rank test. Treatment-related toxicities and recurrence rate were compared using Fisher exact test. All analyses were performed using SPSS ver. 19.0 (IBM, Armonk, NY, USA), and a p-value $<0.05$ was considered statistically significant.

\section{Results}

\section{Patient characteristics}

A total of 126 patients were eligible for analysis. Of them, 74 (58.7\%) were treated with WPRT and concurrent chemotherapy, and 52 (41.3\%) were treated with EF-PRT and concurrent chemotherapy. The majority of the patients (86.5\%) presented with squamous cell carcinoma. The median age was 51 years (range, 23 to 81 years). The largest maximum diameter of primary tumor and short-axis diameter of metastatic pelvic LN in all patients was median $42 \mathrm{~mm}$ (range, 10 to $84 \mathrm{~mm}$ ) and median $12 \mathrm{~mm}$ (range, 6 to $53 \mathrm{~mm}$ ), respectively. Eight patients (6.35\%) had an evidence of common iliac LN metastasis. Among the eight patients, six patients were treated with EFPRT. Patient characteristics of the two treatment groups are given in Table 1.

No significant differences were noted between the groups in terms of patient characteristics, including clinical stage, histological distribution, tumor diameter, metastatic LN size, and level of pretreatment squamous cell carcinoma antigen. Patients who received EF-PRT tended to have a slightly younger age than those who received WPRT, but the difference was not statistically significant $(p=0.066)$. The prescribed radiation dose was also not significantly different in both treatment groups, except the prescribed EBRT dose to the PALN area (Table 2).

\section{Clinical and survival outcomes}

The median follow-up time was 75.5 (range, 5 to 195 months), 
Table 3. Recurrence pattern according to radiation field

\begin{tabular}{lccc}
\multicolumn{1}{c}{ Recurrence pattern } & $\begin{array}{c}\text { Whole pelvic radiotherapy } \\
(\mathrm{n}=74)\end{array}$ & $\begin{array}{c}\text { Extended-field pelvic radiotherapy } \\
(\mathrm{n}=52)\end{array}$ & $\mathrm{p}$-value \\
\hline In-field pelvic recurrence & 11 & 5 & 0.384 \\
Para-aortic lymph node recurrence & 7 & 3 & $0.522^{\text {a) }}$ \\
Distant recurrence & 6 & 8 & 0.201 \\
\hline
\end{tabular}

a) Fisher exact test.

76.5, and 79.3 months for all patients, for patients in the WPRT group, for those in the EF-PRT group, respectively. Treatment failure occurred in 37 patients (29.4\%). Detailed patterns of failure are shown in Table 3. In the EF-PRT group, PALN recurrence was observed in three patients (5.8\%; two isolated out-of-field recurrence and one multiple recurrence of infield pelvic LN and in-field PALN). In the WPRT group, seven patients (9.5\%) developed out-of-field PALN recurrence (five isolated out of field, two multiple recurrence of in-field pelvic LN and in-field PALN). The incidence of PALN recurrence was not significantly different between the treatment groups. The cumulative PALN recurrence rate as classified according to radiation field is shown in Fig. 1. For all patients, the 10-year

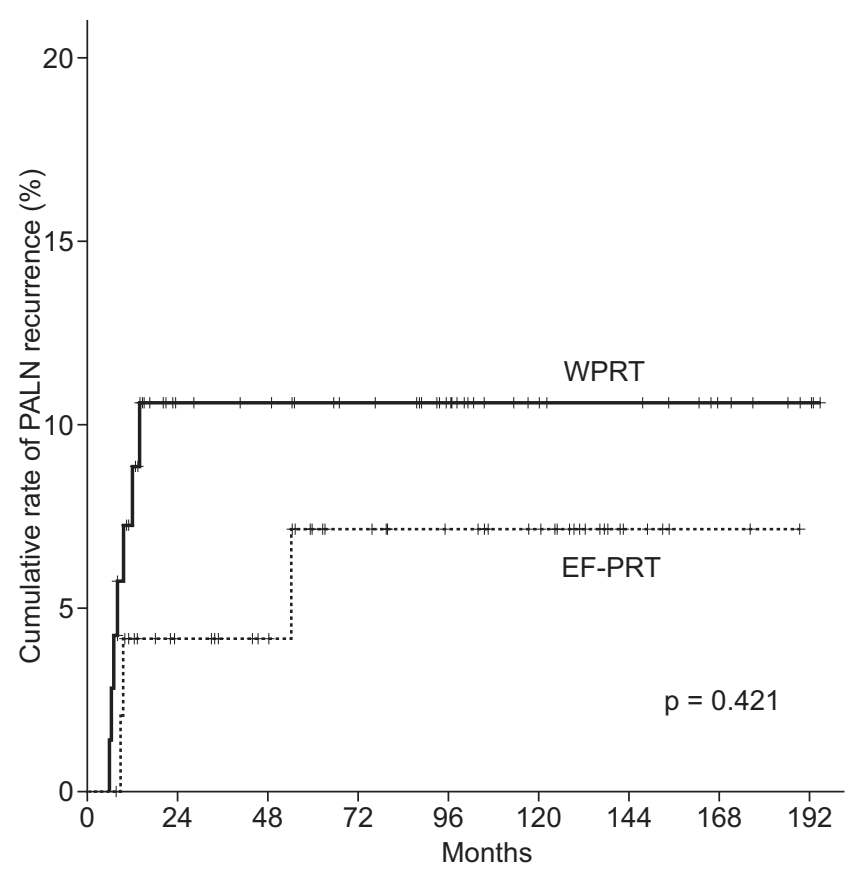

Fig. 1. Cumulative rate of para-aortic lymph node (PALN) recurrence as classified according to the radiotherapy field. The 10-year recurrence rate of PALN involvement: whole pelvic radiotherapy (WPRT) vs. extended-field pelvic radiotherapy (EFPRT), $10.1 \%$ vs. $6.9 \%$, respectively $(p=0.421)$. cumulative PALN recurrence rate for EF-PRT vs. WPRT was 6.9\% vs. $10.1 \%(p=0.421)$, respectively. Fourteen patients $(11.1 \%)$ failed systemically with a total of 16 sites. The most common site of distant metastasis was the lung $(n=9)$, followed by the mediastinal LN $(n=3)$, brain $(n=2)$, liver $(n=1)$, and supraclavicular LN $(n=1)$.

Figs. 2 and 3 illustrate the Kaplan-Meier curves according to the radiation field. The 5- and 10-year DFS for EF-PRT vs. WPRT were $69.7 \%$ vs. $68.1 \%$ and $69.7 \%$ vs. $66.1 \%$ ( $p=0.748$ ), respectively. In addition, the 5- and 10-year OS for EF-PRT vs. WPRT were $77.3 \%$ vs. $75.5 \%$ and $71.7 \%$ vs. $72.3 \%$ ( $p=0.845)$, respectively. The PALN recurrence rate $(p=0.421)$, PFS (logrank, $p=0.748)$, and OS (log-rank, $p=0.845)$ in the WPRT group (Figs. 1-3, Table 3) were not significantly different compared with those in the EF-PRT group.

\section{Toxicity}

A summary of acute and late toxicities is shown in Table 4. The most common acute toxicities were gastrointestinal complications. Acute grade 1-3 gastrointestinal complications were significantly higher in the EF-PRT group ( $n=21 ; 40.4 \%$ ) than in the WPRT group $(n=26 ; 35.1 \%)(p=0.046)$. There were no acute grade 4 complications. Although the difference did not reach statistical significance, the incidence of patients with acute toxicities in the EF-PRT group (26/52 patients, $50 \%)$ was higher than that in the WPRT group (31/74 patients, $41.9 \%)(p=0.368)$. The event of neutropenia was observed 7 patients (9.5\%) in the WPRT group and in 10 patients (19.2\%) in the EF-PRT group, and neutropenia was resolved with medical management. There was no statistically significant difference about the incidence of neutropenia in both treatment groups ( $p=0.114)$. Because of acute complications including neutropenia and/or concerns about a potential toxicity of treatment, 4 patients (5.4\%) in the WPRT group and 10 patients (19.2\%) in the EF-PRT group were treated with reduced dose of chemotherapeutic agent during CCRT. The dose reduction of chemotherapeutic agent during CCRT was observed significantly more in the EF-PRT group than in the 


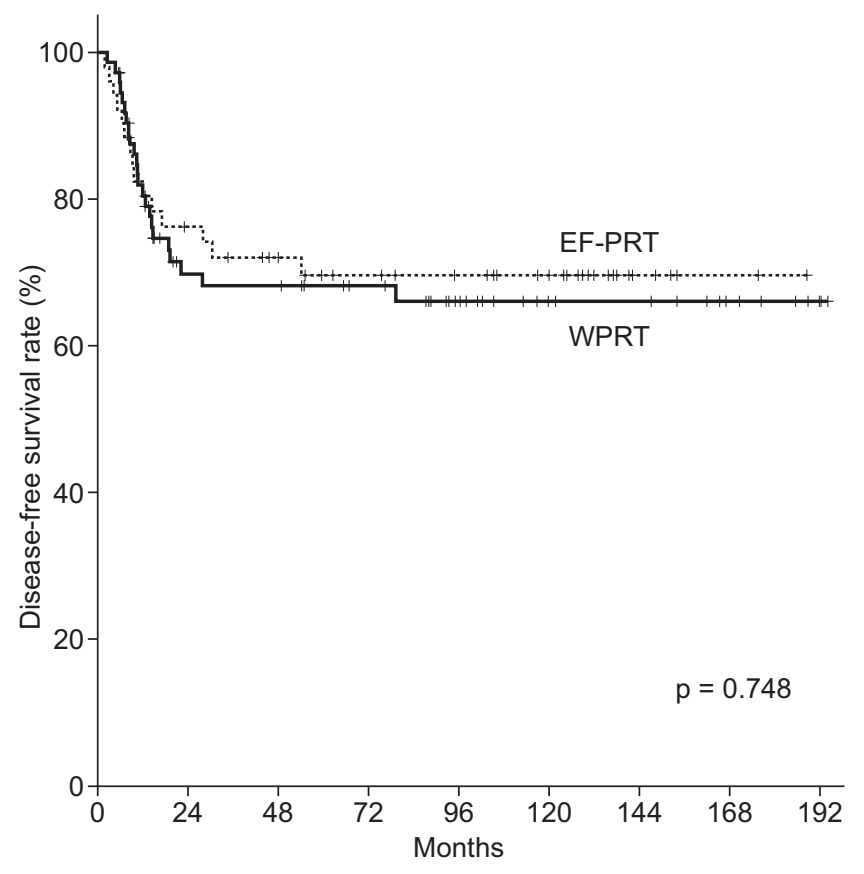

Fig. 2. Disease-free survival estimation using Kaplan-Meier analysis. The 5- and 10-year disease-free survival rate: whole pelvic radiotherapy (WPRT) vs. extended-field pelvic radiotherapy (EF-PRT), $68.1 \%$ and $66.1 \%$ vs. $69.7 \%$ and $69.7 \%$, respectively ( $p=$ 0.748)

WPRT group ( $p=0.015$ ). Due to toxicities that were persistent after dose reduction and poor performance status, only two patients ( 1 in the WPRT group and 1 in the EF-PRT group) stopped chemotherapy. Although overall grade 1-2 acute side effects were often observed during CCRT, they were usually self-limiting or resolved with medical management. In general, WPRT or EF-PRT plus concurrent cisplatin-based chemotherapy was well tolerated. All patients completed the planned EBRT.

Grade 3-4 severe late toxicities were observed in 15 patients $(20.3 \%)$ in the WPRT group and in 15 patients (30.8\%) in the EF-PRT group. Although the absolute percentage of late toxicities was lower in the WPRT group, the difference did not reach statistical significance ( $p=0.178)$. Treatment-related late toxicities in each RT group are summarized in Table 4, and their severity between the treatment groups was not different.

\section{Discussion and Conclusion}

Several randomized studies regarding EF-PRT in the preCCRT era have been published. EF-PRT alone had the potential to reduce PALN recurrence and improve survival. Haie et al. [14] reported the results of the European Organization for

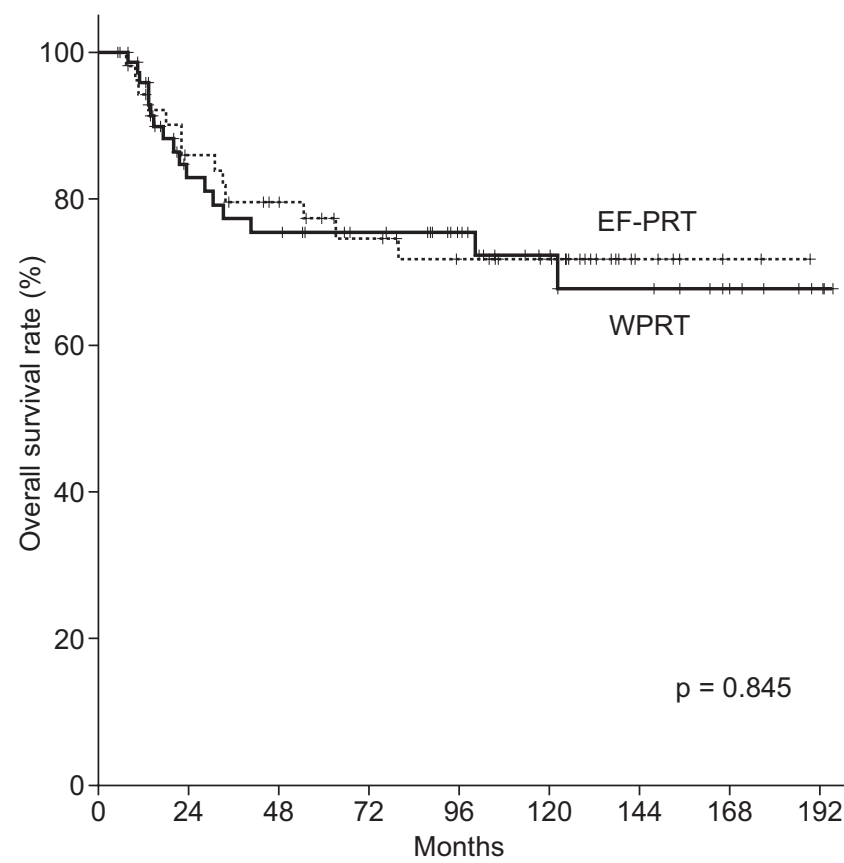

Fig. 3. Overall survival estimation using Kaplan-Meier analysis. The 5- and 10-year overall survival rate: whole pelvic radiotherapy (WPRT) vs. extended-field pelvic radiotherapy (EF-PRT), 75.5\% and $72.3 \%$ vs. $77.3 \%$ and $71.7 \%$, respectively $(p=0.845)$.

Research and Treatment of Cancer Trial that compared the outcomes of two regimens of RT (i.e., EF-PRT vs. WPRT; 45 Gy) in 441 patients with cervical cancer who had no evidence of PALN involvement. No statistically significant difference was found between the two treatment arms with regard to local control, distant metastases, or survival. However, the incidence of para-aortic and distant metastases without pelvic failure was significantly high in patients receiving WPRT. The rate of severe complication was higher at $9 \%$ in patients receiving EFPRT compared with $4.8 \%$ in those receiving WPRT. A similar randomized study was reported by Rotman et al. [12]; they updated the results of the Radiation Therapy Oncology Group (RTOG) randomized trial of 337 cervical cancer patients with no evidence of PALN involvement who were randomized to electively receive or not $45 \mathrm{~Gy}$ to the PALN region. They found a significant difference in OS favoring the EF-PRT arm, although no difference in local recurrence and DFS was noted. The 10-year survival rate was 55\% for patients receiving EFPRT and 44\% for those receiving WPRT ( $p=0.02$ ). The 10-year grade 4 or 5 complication rate was $8 \%$ in the group receiving EF-PRT and $4 \%$ in patients treated with WPRT ( $p=0.06)$. These studies suggested that despite the high complication rates, EF- 
Table 4. Radiation-induced acute and late complications

\begin{tabular}{|c|c|c|c|}
\hline Complication & $\begin{array}{l}\text { Whole pelvic radiotherapy } \\
\qquad(\mathrm{n}=74)\end{array}$ & $\begin{array}{l}\text { Extended field pelvic radiotherapy } \\
\qquad(\mathrm{n}=52)\end{array}$ & p-value \\
\hline Acute complication ${ }^{\text {a) }}$ & $31(41.9)$ & $26(50)$ & 0.368 \\
\hline \multicolumn{4}{|l|}{ Genitourinary } \\
\hline Any & $1(1.4)$ & $4(7.7)$ & $0.152^{c)}$ \\
\hline$\geq$ Grade 3 & $0(0)$ & $0(0)$ & - \\
\hline \multicolumn{4}{|l|}{ Gastrointestinal } \\
\hline Any & $26(35.1)$ & $21(40.4)$ & $0.046^{c)}$ \\
\hline$\geq$ Grade 3 & $2(2.7)$ & $4(7.7)$ & $0.192^{c)}$ \\
\hline \multicolumn{4}{|l|}{ Neutropenia } \\
\hline$\geq$ Grade 3 & 7 (9.5) & 10 (19.2) & 0.114 \\
\hline Late complication ${ }^{\text {b) }}$ & $15(20.3)$ & $16(30.8)$ & 0.178 \\
\hline \multicolumn{4}{|l|}{ Proctitis } \\
\hline Any & $7(9.5)$ & 7 (13.5) & $0.627^{c)}$ \\
\hline$\geq$ Grade 3 & $7(9.5)$ & $7(13.5)$ & $0.627^{\mathrm{c})}$ \\
\hline \multicolumn{4}{|l|}{ Enteritis } \\
\hline Any & $3(4.1)$ & $4(7.7)$ & $0.446^{c)}$ \\
\hline$\geq$ Grade 3 & $1(1.4)$ & $2(3.8)$ & $0.569^{c)}$ \\
\hline \multicolumn{4}{|l|}{ Cystitis } \\
\hline Any & $6(8.1)$ & $7(13.5)$ & 0.331 \\
\hline$\geq$ Grade 3 & $5(6.8)$ & $6(11.5)$ & $0.359^{c)}$ \\
\hline \multicolumn{4}{|l|}{ Rectovaginal fistula } \\
\hline Any & $0(0)$ & $1(1.9)$ & $0.413^{\mathrm{c})}$ \\
\hline$\geq$ Grade 3 & $0(0)$ & $1(1.9)$ & $0.413^{c)}$ \\
\hline \multicolumn{4}{|l|}{ Bowel obstruction } \\
\hline Any & $0(0)$ & $2(3.8)$ & $0.168^{\mathrm{c})}$ \\
\hline$\geq$ Grade 3 & $0(0)$ & $2(3.8)$ & $0.168^{c)}$ \\
\hline \multicolumn{4}{|l|}{ Lymphedema } \\
\hline Any & $3(4.1)$ & $1(1.9)$ & $0.642^{c)}$ \\
\hline$\geq$ Grade 3 & $3(4.1)$ & $1(1.9)$ & $0.642^{c)}$ \\
\hline \multicolumn{4}{|l|}{ Hydronephrosis } \\
\hline Any & $3(4.1)$ & $2(3.8)$ & $1.000^{\mathrm{c})}$ \\
\hline$\geq$ Grade 3 & $3(4.1)$ & $2(3.8)$ & $1.000^{\mathrm{c})}$ \\
\hline
\end{tabular}

Values are presented as number (\%).

Some patients had multiple radiotherapy-induced complications.

a) Total number of patients with concurrent chemoradiotherapy-induced acute complications. ${ }^{\text {b) }}$ Total number of patients with radiotherapy-induced late complications. ${ }^{c}$ Fisher exact test.

PRT had a clinical benefit in the pre-CCRT era.

Since 1999, CCRT has been the standard treatment for locally advanced cervical cancer based on the results of five phase III randomized clinical trials [15-19]. Since then, locally advanced cervical cancer was generally treated with WPRT with concurrent chemotherapy. Although a previous randomized controlled study demonstrated the superiority of WPRT plus concurrent chemotherapy over EF-PRT in patients with stage IB to IIB disease in the setting of definitive RT for cervical cancer $[15,19]$, WPRT plus concurrent chemotherapy has become a standard treatment for this patient population.

Although WPRT was not directly compared with EF-PRT in the CCRT setting, an RTOG 90-01 trial randomized patients with locally advanced cervical cancer to either WPRT with concurrent 5-FU and cisplatin or EF-PRT without concurrent chemotherapy. The study showed that OS and DFS were long in the CCRT arm, with 8-year rates of $41 \%$ and $67 \%$, respectively [19]. These results suggested that the use of concurrent chemotherapy with WPRT to some degree eradicate subclinical metastases beyond the field of RT. Nevertheless, the risk of PALN recurrence remains a concern given that WPRT with concurrent chemotherapy might not eliminate microscopic tumors in the PALN, particularly in patients with risk factors, including positive pelvic LNs. In clinical practice, due to 
concerns regarding toxicity, clinicians also face the dilemma of whether EF-PRT with concurrent chemotherapy should be applied to patients with locally advanced cervical cancer with positive pelvic LNs but negative PALN metastasis as shown on imaging modalities. In line with this, several institutions conducted a retrospective study regarding the feasibility and toxicity of EF-PRT with concurrent chemotherapy, and conflicting evidence regarding acute and late toxicity rates regarding CCRT with EF-PRT were reported [6,20-24].

In the CCRT era, evidence regarding the application of riskbased radiation field (WPRT vs. EF-PRT) for cervical cancer is conflicting. To our knowledge, only three retrospective studies and one prospective study that directly compared WPRT and EF-PRT in the CCRT setting for cervical cancer without evidence of PALN metastasis have been performed since 1999.

Park et al. [25] evaluated the efficacy of EF-PRT in patients with locally advanced cervical cancer without PALN involvement. In their study, CCRT was administered to 133 of 203 patients (62 in EF-PRT, 71 in WPRT), and they reported that in patients treated with CCRT, differences in the treatment field had no significant effect on OS or DFS. Concurrently, Yap et al. [26] reported the results of a retrospective study comparing the outcomes of two regimens of RT in 228 patients (73 patients in the EF-PRT group vs. 155 patients in the WPRT group) with locally advanced cervical cancer with no evidence of PALN involvement. In this study, all patients received external beam WPRT at a median dose of 50 Gy concurrent with weekly cisplatin, followed by a pulsed-dose rate ICBT. The para-aortic area either received or did not receive a median dose of $40 \mathrm{~Gy}$. They reported that the addition of EF-PRT was not associated with a significant difference of DFS or OS on multivariate analysis, and no significant difference in the rate of PALN recurrence was noted in both groups with a median follow-up of 4.6 years. Among the 228 patients, the pelvic LN-positive cohort comprised 67 patients (29.4\%; 46 patients in the EF-PRT treatment group and 21 patients in the WPRT treatment group). Looking at this subgroup separately, the addition of PALN RT had also no significant effect on DFS (hazard ratio $[\mathrm{HR}], 1.05$; confidence interval [CI], 0.56-1.99; $p=$ 0.95), OS (HR, 0.98; $\mathrm{Cl}, 0.42-2.29 ; \mathrm{p}=0.96)$, or PALN recurrence rate $(\mathrm{HR}, 2.01 ; \mathrm{Cl}, 0.79-5.12 ; \mathrm{p}=0.21)[26]$.

Meanwhile, other studies have reported the potential benefit of EF-PRT with concurrent chemotherapy. In a small randomized, prospective study, Asiri et al. [27] showed that prophylactic EF-PRT with concurrent chemotherapy can be a reasonable option for patients with locally advanced cervical cancer with radiologically positive pelvic LN and radiographically negative PALN. Recently, Lee et al. [28] reported a prophylactic lower para-aortic RT using IMRT with weekly cisplatin for FIGO stage IB2-IVA cervical cancer with negative PALN involvement. They reported that although the clinical outcome for patients with FIGO IBIIB and negative pelvic LN was not significantly different, the 5-year PALN recurrence-free survival and OS for WPRT vs. EF-PRT were $80.1 \%$ vs. $96.4 \%(p=0.02)$ and $58.1 \%$ vs. $83.5 \%(p=0.012)$, respectively, in patients with stage III-IVA or positive pelvic LN. They concluded that EF-PRT with IMRT and concurrent chemotherapy reduced PALN recurrence and improved OS without increasing the occurrence of severe toxicities for patients with locally advanced cervical cancer and positive pelvic LN or FIGO III-IVA disease. However, these nonrandomized studies had heterogeneity in tumor characteristics, and the patients in the EF-PRT group had more advanced disease in terms of tumor stage and positive pelvic LNs than those in the WPRT group. In the prospective study, they also had poor randomization, selection bias, and low sample size [27]. Thus, in patients undergoing platinum-based CCRT, the role of EF-PRT has not been clearly established, particularly in the presence of pelvic LN involvement.

In this study, we assessed PALN recurrence rate, DFS, and OS in patients with pelvic LN-positive cervical cancer treated with concurrent chemotherapy plus EF-PRT or WPRT. Seven (9.46\%) and $3(5.77 \%)$ patients had PALN recurrence in the WPRT group and EF-PRT group $(p=0.522)$, respectively, and the 10 year cumulative PALN recurrence rate as classified according to the radiation field for the WPRT vs. EF-PRT was $10.1 \%$ vs. $6.9 \%$ $(p=0.421)$, respectively. Although a relatively high incidence of PALN failure was shown, the findings did not have statistical significance. As shown in Figs. 2 and 3, when the patients were treated with EF-PRT plus concurrent chemotherapy, no significant difference was noted in DFS and OS, indicating that the addition of EF-PRT to WPRT plus concurrent chemotherapy did not have a significant impact on the clinical outcome. We found that patients with pelvic LN-positive cervical cancer treated with cisplatin-based CCRT did not benefit from prophylactic EF-PRT. Our findings are consistent with those of Park et al. [25] and Yap et al. [26]. A recent meta-analysis regarding EF-PRT by Sapienza et al. [29] reported that cancerrelated death was not significantly altered (odds ratio, 0.68; $\mathrm{Cl}, 0.45-1.01 ; p=0.06$ ), and the potential impact of PALN RT on survival warrants a reconsideration of EF-PRT. Collectively, these results of the present and previous studies indicate that the clinical benefit of EF-PRT with concurrent chemotherapy was limited. 
The present study has some limitations. Our study was a retrospective analysis of the data from a single institution and thus could have a selection bias. Treatment strategy in terms of chemotherapy regimen and radiation field (the upper border of EF-PRT or WPRT) was not consistently controlled in all patient cohort. A relatively small patient number is also a weak point. Another limitation is retrospective data collection on complication. Based on all the medical records available in our institution, the toxicity results were described. Because this study was retrospective in nature and medical records were incomplete, we may have underestimated the toxicities. However, several important findings were obtained. The study was intended solely for cervical cancer patients with evidence of pelvic LN involvement. Although the study design was retrospective, patient characteristics in the two groups were not significantly different. The follow-up period was also appropriate. Based on these factors, this study provides additional evidence supporting the use of WPRT rather than EF-PRT in CCRT for cervical cancer with the evidence of pelvic LN involvement.

In conclusion, the present study suggests that EF-PRT does not have an additional benefit in patients with pelvic LNpositive cervical cancer treated with CCRT. Although pelvic LN involvement has a risk of occult PALN metastasis, no benefits of prophylactic PALN RT with concurrent cisplatinbased chemotherapy were noted. A well-designed prospective randomized study with a large patient cohort and multiinstitutional participation is necessary to determine whether or not EF-PRT with concurrent chemotherapy improves the clinical outcomes compared with WPRT with concurrent chemotherapy and to evaluate the toxicity of EF-PRT with concurrent chemotherapy, particularly in patients with pelvic LN involvement.

\section{Conflict of Interest}

No potential conflict of interest relevant to this article was reported.

\section{References}

1. Sevin BU, Lu Y, Bloch DA, Nadji M, Koechli OR, Averette HE. Surgically defined prognostic parameters in patients with early cervical carcinoma: a multivariate survival tree analysis. Cancer 1996;78:1438-46.

2. Yalman $D$, Aras $A B$, Ozkok $S$, et al. Prognostic factors in definitive radiotherapy of uterine cervical cancer. Eur J
Gynaecol Oncol 2003;24:309-14.

3. Tseng JY, Yen MS, Twu NF, et al. Prognostic nomogram for overall survival in stage IIB-IVA cervical cancer patients treated with concurrent chemoradiotherapy. Am J Obstet Gynecol 2010;202:174.e1-7.

4. Kidd EA, Siegel BA, Dehdashti $F$, et al. Lymph node staging by positron emission tomography in cervical cancer: relationship to prognosis. J Clin Oncol 2010;28:2108-13.

5. Berman ML, Keys H, Creasman W, DiSaia P, Bundy B, Blessing J. Survival and patterns of recurrence in cervical cancer metastatic to periaortic lymph nodes (a Gynecologic Oncology Group study). Gynecol Oncol 1984;19:8-16.

6. Ring KL, Young JL, Dunlap NE, Andersen WA, Schneider BF. Extended-field radiation therapy with whole pelvis radiotherapy and cisplatin chemosensitization in the treatment of IB2-IIIB cervical carcinoma: a retrospective review. Am J Obstet Gynecol 2009;201:109.e1-6.

7. Sakuragi N, Satoh C, Takeda N, et al. Incidence and distribution pattern of pelvic and paraaortic lymph node metastasis in patients with Stages IB, IIA, and IIB cervical carcinoma treated with radical hysterectomy. Cancer 1999;85:1547-54.

8. Michel G, Morice P, Castaigne D, Leblanc M, Rey A, Duvillard P. Lymphatic spread in stage Ib and II cervical carcinoma: anatomy and surgical implications. Obstet Gynecol 1998;91:360-3.

9. Lagasse LD, Creasman WT, Shingleton HM, Ford JH, Blessing $J A$. Results and complications of operative staging in cervical cancer: experience of the Gynecologic Oncology Group. Gynecol Oncol 1980;9:90-8.

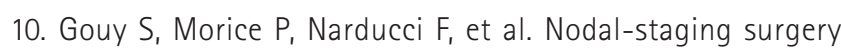
for locally advanced cervical cancer in the era of PET. Lancet Oncol 2012;13:e212-20.

11. Rotman M, Choi K, Guse C, Marcial V, Hornback N, John M. Prophylactic irradiation of the para-aortic lymph node chain in stage IIB and bulky stage IB carcinoma of the cervix, initial treatment results of RTOG 7920. Int J Radiat Oncol Biol Phys 1990;19:513-21.

12. Rotman $M$, Sedlis $A$, Piedmonte $M R$, et al. A phase III randomized trial of postoperative pelvic irradiation in Stage IB cervical carcinoma with poor prognostic features: follow-up of a gynecologic oncology group study. Int J Radiat Oncol Biol Phys 2006;65:169-76.

13. Joiner $M$, Bentzen $S$. Fractionation: the linear-quadratic approach. In: Joiner $M$, van der Kogel A, editors. Basic clinical radiobiology. 4th ed. London: Hodder Arnold; 2009. p. 102-33.

14. Haie C, Pejovic MH, Gerbaulet A, et al. Is prophylactic paraaortic irradiation worthwhile in the treatment of advanced 
cervical carcinoma? Results of a controlled clinical trial of the EORTC radiotherapy group. Radiother Oncol 1988;11:101-12.

15. Morris M, Eifel PJ, Lu J, et al. Pelvic radiation with concurrent chemotherapy compared with pelvic and para-aortic radiation for high-risk cervical cancer. N Engl J Med 1999;340:1137-43.

16. Rose PG, Bundy BN, Watkins EB, et al. Concurrent cisplatinbased radiotherapy and chemotherapy for locally advanced cervical cancer. N Engl J Med 1999;340:1144-53.

17. Whitney CW, Sause W, Bundy BN, et al. Randomized comparison of fluorouracil plus cisplatin versus hydroxyurea as an adjunct to radiation therapy in stage IIB-IVA carcinoma of the cervix with negative para-aortic lymph nodes: a Gynecologic Oncology Group and Southwest Oncology Group study. J Clin Oncol 1999;17:1339-48.

18. Wong LC, Ngan HY, Cheung AN, Cheng DK, Ng TY, Choy DT. Chemoradiation and adjuvant chemotherapy in cervical cancer. J Clin Oncol 1999;17:2055-60.

19. Eifel PJ, Winter $K$, Morris $M$, et al. Pelvic irradiation with concurrent chemotherapy versus pelvic and para-aortic irradiation for high-risk cervical cancer: an update of radiation therapy oncology group trial (RTOG) 90-01. J Clin Oncol 2004;22:872-80.

20. Malfetano JH, Keys H, Cunningham MJ, Gibbons S, Ambros R. Extended field radiation and cisplatin for stage IIB and IIIB cervical carcinoma. Gynecol Oncol 1997;67:203-7.

21. Chung YL, Jian JJ, Cheng SH, et al. Extended-field radiotherapy and high-dose-rate brachytherapy with concurrent and adjuvant cisplatin-based chemotherapy for locally advanced cervical cancer: a phase I/II study. Gynecol Oncol 2005;97:126-35.

22. Gerszten $K_{1}$ Colonello K, Heron DE, et al. Feasibility of concurrent cisplatin and extended field radiation therapy (EFRT) using intensity-modulated radiotherapy (IMRT) for carcinoma of the cervix. Gynecol Oncol 2006;102:182-8.

23. Uno $T$, Mitsuhashi $A$, Isobe $K$, et al. Concurrent daily cisplatin and extended-field radiation therapy for carcinoma of the cervix. Int J Gynecol Cancer 2008;18:80-4.

24. Grigsby PW, Heydon K, Mutch DG, Kim RY, Eifel P. Long-term follow-up of RTOG 92-10: cervical cancer with positive paraaortic lymph nodes. Int J Radiat Oncol Biol Phys 2001;51:9827.

25. Park SG, Kim JH, Oh YK, et al. Is Prophylactic irradiation to para-aortic lymph nodes in locally advanced cervical cancer necessary? Cancer Res Treat 2014;46:374-82.

26. Yap ML, Cuartero J, Yan J, et al. The role of elective para-aortic lymph node irradiation in patients with locally advanced cervical cancer. Clin Oncol (R Coll Radiol) 2014;26:797-803.

27. Asiri MA, Tunio MA, Mohamed R, et al. Is extended-field concurrent chemoradiation an option for radiologic negative paraaortic lymph node, locally advanced cervical cancer? Cancer Manag Res 2014;6:339-48.

28. Lee J, Lin JB, Chang CL, et al. Prophylactic lower para-aortic irradiation using intensity-modulated radiotherapy mitigates the risk of para-aortic recurrence in locally advanced cervical cancer: a 10-year institutional experience. Gynecol Oncol 2017:146:20-6.

29. Sapienza LG, Gomes MJ, Calsavara VF, Leitao MM Jr, Baiocchi G. Does para-aortic irradiation reduce the risk of distant metastasis in advanced cervical cancer? A systematic review and meta-analysis of randomized clinical trials. Gynecol Oncol 2017;144:312-7. 